\title{
A Student Control Competition Through a Remote Robotics Lab
}

\author{
A new tool for demonstrating \\ control design issues
}

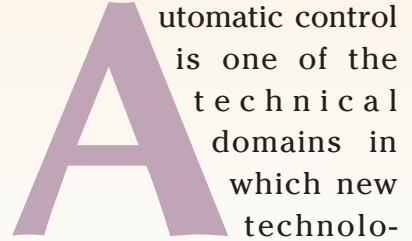

gies and tools for distance learning have often been applied [1]. The Automatic Control Telelab (ACT) is a remote laboratory for automatic control developed at the University of Siena. This article describes a new ACT module referred to as the student competition system.

ACT has been used in undergraduate control systems courses since 1999 [2]. The aim of the project is to allow students to apply their knowledge of control theory in an easy way, without restrictions due to laboratory operating times and process availability. Currently, ACT is accessible 24 hours a day from any computer connected to the Internet. Key features of ACT are its simple user-friendly interface and the possibility of integrating user-defined controllers in the control loop of the remote process through a

\section{By Marco Casini, Domenico} Prattichizzo, and Antonio Vicino
MATLAB/Simulink model. ACT is continually upgraded with new software versions and experiments. In the present ACT release, five processes are available for online experiments: a dc motor, a tank, a magnetic levitation system, a two-degreeof-freedom helicopter, and a Lego Mindstorms mobile robot [3].

A new component of the ACT experience is a student competition, in which students or groups of students compete to develop the best controller for a given remote process. A typical competition session starts with defining control system requirements for one of the ACT processes. Students then access ACT and design controllers to steer the process during the competition. The ACT server stores the students' controllers and data in a database, computes the performance measures, and assigns a score to the controllers. A ranking is computed and reported to students as feedback for their learning process. 


\section{Student Competition Overview}

Most remote labs allow users to run experiments using predefined or user-defined controllers. Students can run an experiment and look at the dynamic response, but information on the controller's performance is not generally provided, and it is not possible to know how controllers designed by other people behave on the same process. This deficiency is one of our main motivations for designing a student competition for the remote laboratory. Through this tool, students are aware of the performance requirements that their controllers must satisfy. Moreover, a ranking of the best controllers, as well as the time plots of related experiments, are provided.

Features of the ACT competition system are described in the following sections.

\section{Remote Exercises}

In addition to controller synthesis exercises, this tool challenges students to design a controller that must satisfy performance requirements and allows them to test their controller on a remote real process. At the end of the experiment, the performance measures are automatically computed and shown to the user; if such measures fulfill the requirements, the exercise is completed. An overall measure, usually a weighted sum of the previous measures, is then computed, and the controller is included in the ranking list.

\section{Controller Comparison}

The competition ranking is provided to the students. During the final competition lesson, students who have designed the best controllers are invited to discuss their projects, while the instructor explains why some control strategies work better than others.

\section{Many Benchmarks on the Same Process}

It is possible to provide more than one competition benchmark on the same process, thus increasing the number of remote exercises available for students. New benchmarks can easily be added for implementation owing to the software architecture of the ACT competition system.

\section{A Competition Session Description}

In this section, we describe a competition session involving the magnetic levitation process. The requirements for a step reference input are as follows:

- the $5 \%$ settling time must be less than 1 second

- the time response overshoot must be less than $10 \%$. To compete, students register by completing a form and subsequently receive a username and password. The suc- cessive steps during a competition session are summarized as follows:

- study the physical model of the process and linearize it around an operating point

- analyze the linear model through frequency domain plots and root locus techniques to determine appropriate bounds for the controller gains
- synthesize a controller that achieves the given performance specifications by using lead-lag or proportional-integral differential (PID) controllers

- construct a Simulink model of the controller

- simulate the controller

- test the controller on the actual remote process

- analyze the system response and, if not satisfactory, adjust the controller and try again.

To design the controller, students must download a Simulink template file and connect the signals describing the reference, output, and command signals with suitable Simulink blocks. Since the output signal is the sensor reading, the students must insert a block that models the sensor characteristic.

A special graphical interface allows students to describe the structure of their controllers, such as PID, and set the sample time of the experiment. In addition, the user must specify the file containing the controller and, if needed, the MATLAB workspace file (.mat) containing the data for his/her controller. These files are uploaded to the server, compiled, and, if no error occurs, executed on the real remote process. A second graphical interface (Figure 1) allows the user to start the experiment and observe its behavior through plots and live video windows. At the end of the experiment, the performance measures are automatically computed and displayed.

Since different controllers can satisfy the performance specifications, an overall score is computed to generate a ranking (Figure 2). This overall score is obtained as a weighted sum of the performance measures. If a controller does not satisfy the performance specifications, an overall score is not computed.

It is possible for each user to view a controller report with information on ranking and other data, such as the controller description, the nickname of the user, and the user's institution. 


\section{Teaching Experiences}

In this section, we describe the role of the student competition in undergraduate control system classes at the University of Siena. The lecturer begins by illustrating the
At the end of the competition, most of the students are able to design a satisfactory controller. Student feedback on the project has been enthusiastic, especially since students are able to apply many theoretical notions. Moreover, the students try to do their best to obtain a good ranking. However, the real motivation for the competition is not merely to determine a winner but rather to give students a new tool that can help them better understand practical control design issues, while increasing their interest in control systems and technology.

\section{remote process through a MATLAB/Simulink model.}

physical model of the magnetic levitation system, emphasizing its unstable and nonlinear dynamics, and suggesting how the students might linearize the dynamics to design the controller.

Since ACT is accessible at all times, unless being used by someone else, students are able to analyze the system, design candidate controllers, and test them prior to the second competition class. During the second class, the lecturer answers student questions and helps solve typical problems. For example, the lecturer might suggest the use of a prefilter on the reference input to obtain smoother command signals and, as a result, better performance.

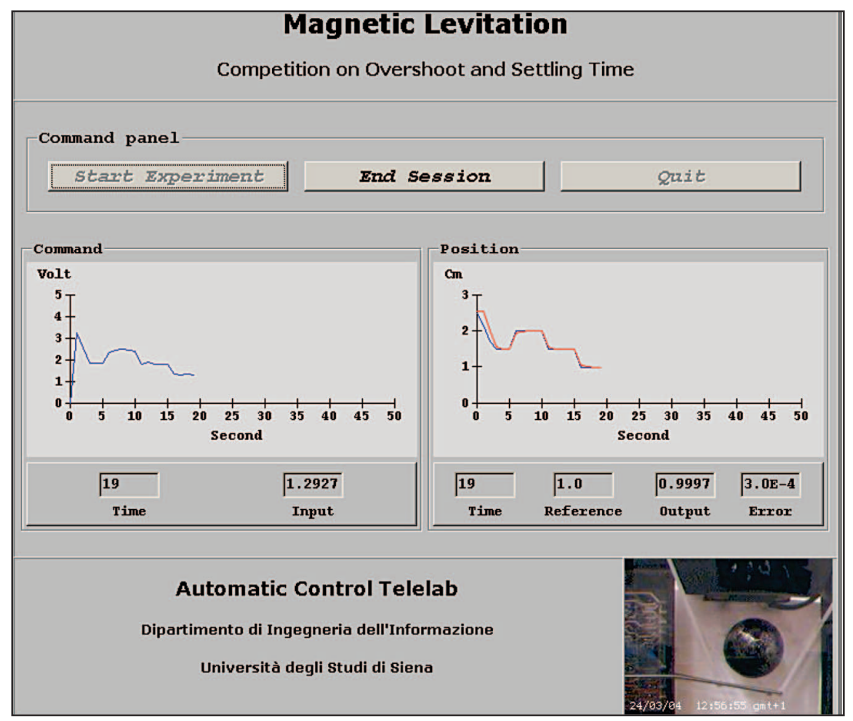

Figure 1. The interface showing the running experiment. Through this interface, it is possible to start and stop the experiment, as well as view data plots and online video.

\section{Conclusions}

In this article, the student competition of ACT is described. Control systems classes at the University of Siena are using the ACT competition system with great interest and excitement. This tool stimulates students' interest in control systems and enhances learning by facilitating the understanding of practical control design issues. We are currently designing an ACT competition for a Lego mobile robot, which can be used by students of robotics courses. The ACT home page is http://act.dii.unisi.it.

\section{References}

[1] S.E. Poindexter and B.S. Heck, "Using the Web in your courses: What can you do? What should you do?," IEEE Contr. Syst. Mag., vol. 19, no. 1, pp. 83-92, 1999.

[2] M. Casini, D. Prattichizzo, and A. Vicino, "The Automatic Control Telelab: A user-friendly interface for distance learning," IEEE Trans. Educ., vol. 46, no. 2, pp. 252-257, 2003.

[3] M. Casini, D. Prattichizzo, and A. Vicino, "The Automatic Control Telelab. A Web-based technology for distance learning," IEEE Contr. Syst. Mag., vol. 24, no. 3, pp. 36-44, 2004.

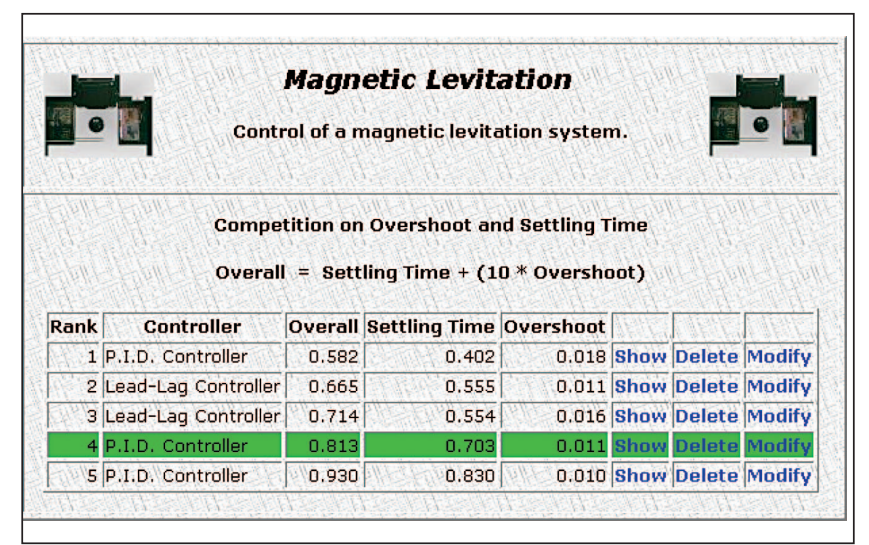

Figure 2. Controller ranking. At the end of the experiment, performance measures are automatically computed, and the controller is included in the ranking list. 
Marco Casini (casini@ing.unisi.it) received the Laurea degree in computer science engineering and the Ph.D. in control systems from the University of Siena in 1999 and 2003, respectively. He is currently a research associate at the Dipartimento di Ingegneria dell'Informazione of the University of Siena. Since 1999, he has held several fellowships at the Dipartimento di Ingegneria dell'Informazione of the University of Siena. In 2001, he was a visiting scientist at the Laboratory for Information and Decision Systems (LIDS) at the Massachusetts Institute of Technology, Cambridge. His research interests include distance learning, remote laboratories, set membership identification, and identification for control. He can be contacted at the Dipartimento di Ingegneria dell'Informazione, Università degli Studi di Siena, Via Roma 56, 53100 Siena, Italy.

Domenico Prattichizzo received the M.S. degree in electronics engineering and the Ph.D. degree in robotics and automation from the University of Pisa in 1991 and 1995 , respectively. In 1994, he was visiting scientist at the Artificial Intelligence Lab at the Massachusetts Institute of Technology. Since 2002, he has been an associate professor at the University of Siena. He was cochair of the Second IEEE International Workshop on Control Problems in Robotics and Automation in 2002. His research activities include distance learning, optimal control, robotic manipulation, and visual servoing. He is an associate editor of
IEEE Transactions on Robotics and IEEE Transactions on Control System Technology, an editorial board member of the Journal of Dynamics of Continuous, Discrete and Impulsive Systems Series $B$, and a member of the Conference Editorial Board of the IEEE Control Systems Society. He is coauthor of more than 100 papers in the area of control systems and robotics.

Antonio Vicino received the Laurea in electrical engineering from the Politecnico di Torino, Torino, Italy. From 1987 to 1990 , he was associate professor of control systems at the Università di Firenze. In 1990, he joined the Dipartimento di Ingegneria Elettrica, Università di L'Aquila, as professor of control systems. Since 1993, he has been with the Università di Siena, where he is currently dean of the engineering faculty. In 2000, he founded the Center for Complex Systems Studies of the University of Siena, where he is currently director. He was an associate editor for IEEE Transactions on Automatic Control from 1992 to 1996. Presently, he is an associate editor for Automatica and associate editor at large for IEEE Transactions on Automatic Control. He is a Fellow of the IEEE and author of 190 technical publications. He is also coeditor of two books on robustness in identification and control. His main research interests are in robust control of uncertain systems, robust identification and filtering, applied system modeling, and mobile robotics.

\section{Job Advancement}

A classic example is said to be that of Humphrey Potter, a twelve-year-old boy employed in the early eighteenth century in England to operate a handle that admitted steam to the cylinder of Newcomen's first steam engine. Potter, being a person of considerable intelligence, yet having nothing else to do but open and close steam valves, noted that whenever the piston was at one end of the cylinder he opened one valve, and whenever it was at the other end he opened another to let the steam into the other side of the piston. He observed the piston-valve relationship, hooked the valve to the piston so that the piston operated the valve automatically and thereby invented the slide valve mechanism which is still in use today. Incidentally he worked himself out of a job and into a better one.

- Quoted from W. Buckingham, Automation, Its Impact on Business and People, Harper, 1961. 